\title{
Average auroral configuration parameterized by geomagnetic activity and solar wind conditions
}

\author{
S. E. Milan ${ }^{1}$, T. A. Evans ${ }^{1}$, and B. Hubert ${ }^{2}$ \\ ${ }^{1}$ Department of Physics and Astronomy, University of Leicester, Leicester LE1 7RH, UK \\ ${ }^{2}$ Laboratory of Planetary and Atmospheric Physics, University of Liége, Liége, 4000 Belgium \\ Received: 15 January 2009 - Revised: 9 March 2010 - Accepted: 19 April 2010 - Published: 22 April 2010
}

\begin{abstract}
Average proton and electron auroral images are compiled from three years of observations by the IMAGE spacecraft, binned according to concurrent $K_{P}$ and upstream solar wind conditions measured by the ACE spacecraft. The solar wind parameters include solar wind velocity, density, and pressure, interplanetary magnetic field (IMF) magnitude and orientation, and an estimate of the magnetopause reconnection rate. We use both (a) the overall variation in brightness in the images and (b) the variation in location of the aurorae with respect to the binning parameters to determine which parameters best order the auroral response. We find that the brightness varies by a factor of $\sim 50$ with $K_{P}$, a similar amount with estimated dayside reconnection voltage, $\sim 15$ with the IMF, $\sim 3$ with solar wind density, $\sim 2$ with solar wind velocity, and $\sim 5$ with pressure. Clearly, geomagnetic activity as measured by $K_{P}$ and auroral dynamics are closely associated. In terms of the solar wind-magnetosphere coupling that drives auroral dynamics, the IMF is of paramount importance in modulating this, with solar wind speed and density playing a lesser role. Dayside reconnection voltage, derived from the solar wind velocity and IMF magnitude and orientation, orders the data almost as well as $K_{P}$, though we find a plateau in the auroral response between voltages of 100 and $150 \mathrm{kV}$. We also discuss changes in configuration and overall size of the average auroral oval with upstream conditions.
\end{abstract}

Keywords. Magnetospheric physics (Auroral phenomena; Magnetospheric configuration and dynamics; Solar windmagnetosphere interactions)

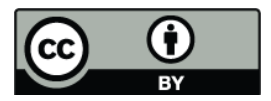

Correspondence to: S. E. Milan (steve.milan@ion.le.ac.uk)

\section{Introduction}

Feldstein and Starkov (1967) used photographs of the aurorae to determine the average locations of the poleward and equatorward edges of the auroral zone for differing levels of geomagnetic activity, parameterized by the magnetic activity index $Q$. Subsequently, Holzworth and Meng (1975) represented the locations of these boundaries by truncated Fourier series to simplify their calculation. The auroral zone was shown to extend to lower latitudes and to increase in latitudinal width during periods of geomagnetic disturbance when $Q$ was enhanced. This auroral zone was thought of as the region of maximum probability of observation of auroral forms; with the advent of the Space Age and the launch of spacecraft with cameras onboard able to make global observations of the aurorae, such as Dynamics Explorer 1 (Frank and Craven, 1988), it was confirmed that the auroral oval was usually present as a coherent feature encircling the poles.

Since that time, there has been considerable study of the location and intensity of the aurorae - or the precipitating particles responsible for the formation of the aurorae - for differing levels of geomagnetic activity, and of the response of the aurorae to changing up-stream solar wind conditions. For instance, Hardy et al. $(1985,1989)$ and Gussenhoven et al. (1987) used polar orbiting spacecraft to examine the spatial patterns of the precipitating flux and average energy of auroral electrons and protons, again parameterized by a geomagnetic index, in this case $K_{P}$. More recently, with the availability of large datasets of global auroral imaging from the Polar and IMAGE spacecraft, investigation of the average configuration of the auroral oval and the response of the precipitating energy flux to changing up-stream solar wind conditions has been possible (e.g., Liou et al., 1998; Shue et al., 2002; Coumans et al., 2004, 2006, 2007). Such studies are akin to others examining the magnetospheric response to

Published by Copernicus Publications on behalf of the European Geosciences Union. 
differing solar wind driving conditions, where that response is measured by, for instance, a variety of geomagnetic activity indices (e.g., Fairfield, 1967; Scurry and Russell, 1991; Newell et al., 2007), cross polar cap potential (e.g., Reiff et al., 1981), or ionospheric convection (e.g., Heppner and Maynard, 1987; Ruohoniemi and Greenwald, 1996). Determination of which parameters best order the magnetospheric response gives insights into the coupling mechanisms that feed solar wind energy into the magnetosphere. In this study we produce maps of the average electron and proton auroral configuration for differing geomagnetic conditions from three years of observations by the Imager for Magnetopauseto-Aurora Global Exploration (IMAGE) spacecraft (Burch et al., 2000). We parameterize these maps by $K_{P}$ and by upstream solar wind conditions determined by the Advanced Composition Explorer (ACE; Stone et al., 1998), including solar wind speed, density, and pressure, the orientation of the interplanetary magnetic field (IMF), and the solar wind electric field. In this way we can determine which factors have the most impact on auroral configuration and intensity.

\section{Observations}

The IMAGE mission provided approximately 5.5 years of observations of the Northern and Southern Hemisphere UV aurorae, between 2000 and 2005, using the Far Ultraviolet (FUV) instrument (Mende et al., 2000a,b) which comprised the Wideband Imaging Camera (WIC) and the Spectrographic Imager (SI). WIC was sensitive to auroral emissions over a broad range of the UV spectrum, the primary component of which is associated with precipitating electrons. The SI12 channel imaged Doppler-shifted Lyman $\alpha$ emission associated with precipitating protons. The orbital geometry and spin of the IMAGE spacecraft was such that images were captured with a cadence of 2 min for approximately $10 \mathrm{~h}$ of each $14 \mathrm{~h}$ orbit.

For this study, we produced average auroral maps that are constructed from images of the Northern or Southern Hemisphere auroral oval. Precession of the apogee of the orbit of IMAGE meant that the Northern Hemisphere auroral oval was imaged best during the first portion of the mission, and we chose to focus on the 2-year period June 2000 to May 2002. Due to contamination of the WIC observations by dayglow we limited study of WIC images to the five months straddling northern winter solstice: October, November, December, January, and February. Our Northern Hemisphere dataset thus comprised close to 350000 images from SI12 and 150000 images from WIC. The Southern Hemisphere auroral oval was imaged best between January 2004 and September 2005, though instrumental issues meant that we had to disregard observations from the months March to $\mathrm{Au}-$ gust 2004 and July and August 2005, leaving just over 13 months of data from the SI12 camera. In addition, we were left with too few observations from austral winter to make analysis of the WIC images worthwhile. Hence, our Southern Hemisphere dataset comprised just over 150000 SI12 images.

This primary dataset was pre-processed to speed up subsequent analysis. Each image was mapped into a magnetic latitude and MLT coordinate system and then re-binned into a $40 \times 40$ Cartesian grid of cells $222 \times 222 \mathrm{~km}$ in size $(222 \mathrm{~km}$ is equivalent to $2^{\circ}$ of latitude), centred on the geomagnetic pole with rows and columns aligned along the noon-midnight and dawn-dusk meridians. The image intensities are recorded as counts, which have been flat-fielded and corrected for the gain of the detector. Although not physical units, the counts scale linearly with emission intensity. For instance, Frey et al. (2003) estimated the emission intensity for SI12 as $1 \mathrm{~mW} \mathrm{~m} \mathrm{~m}^{-2}$ for 30 and 17 counts pixel ${ }^{-1} \mathrm{~s}^{-1}$ for assumed incident proton energies of 8 and $25 \mathrm{keV}$, respectively. In what follows, all SI12 observations are shown on the same colour scale. A different scale is used for WIC images.

The Northern Hemisphere IMAGE observations were binned according to integer values of $K_{P}$ and the individual images summed to produce average images for each $K_{P}$ level. The results for SI12 and WIC are shown in Fig. 1. Each panel shows the average intensity in a magnetic latitude and magnetic local time polar projection, with local noon at the top of each image. White radial lines show meridians of MLT and white circles are magnetic latitudes in steps of $10^{\circ}$. For $K_{P} \leqslant 4$, the corresponding Feldstein and Starkov (1967) auroral oval, calculated from the model of Holzworth and Meng (1975), has been superimposed. In the top right of each panel is the number of images that contributed to the average image. A contribution from dayglow can be seen at the top of each image, especially in the case of WIC. In addition to dayglow, each image has a non-zero background level which is assumed to be uniform across the images; this is removed when comparing brightnesses between the images.

Similarly, average images were produced by binning with respect to observations of the solar wind by ACE located approximately $230 R_{\mathrm{E}}$ upstream of the Earth. Solar wind density, velocity, and pressure measurements were provided by the Solar Wind Electron, Proton and Alpha Monitor (SWEPAM) instrument (McComas et al., 1998) and IMF measurements by the MAG instrument (Smith et al., 1998). ACE observations were lagged to the magnetopause by considering the solar wind velocity. Average images binned according to IMF clock angle and IMF magnitude $|B|$ in steps of $45^{\circ}$ and $5 \mathrm{nT}$ are presented in Fig. 2 (upper panels) for SI12. For brevity, and due to the presence of dayglow, we don't show the corresponding WIC images. The bottom panels of Fig. 2 show the SI12 auroral observations binned by solar wind electric field $E_{\mathrm{SW}}=V_{\mathrm{SW}} \sqrt{B_{Y}^{2}+B_{Z}^{2}} \sin ^{2} \frac{1}{2} \theta$ (Kan and Lee, 1979). We have multiplied $E_{\mathrm{SW}}$ by $2.75 R_{\mathrm{E}}$, where $R_{\mathrm{E}}$ is the radius of the Earth, to convert the solar wind electric field to an estimated low-latitude magnetopause reconnection voltage, $\Phi_{D} ; 2.75 R_{\mathrm{E}}$ is an estimate of the effective length 

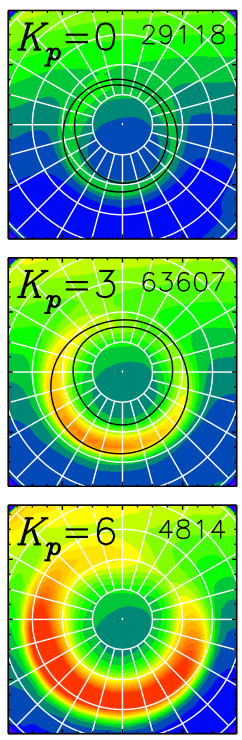

SI12 counts 1000
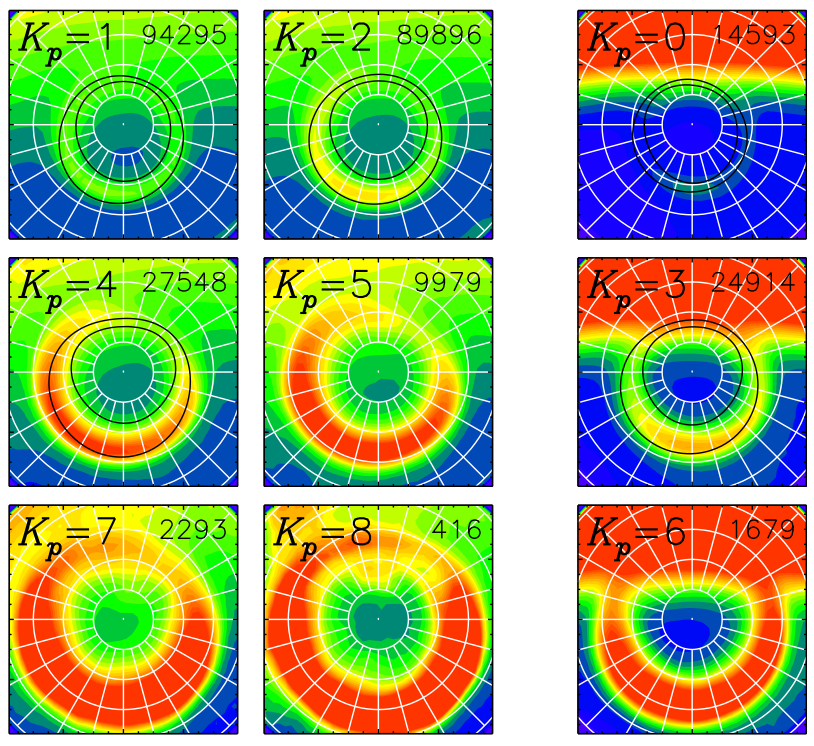

500 WIC counts 2500
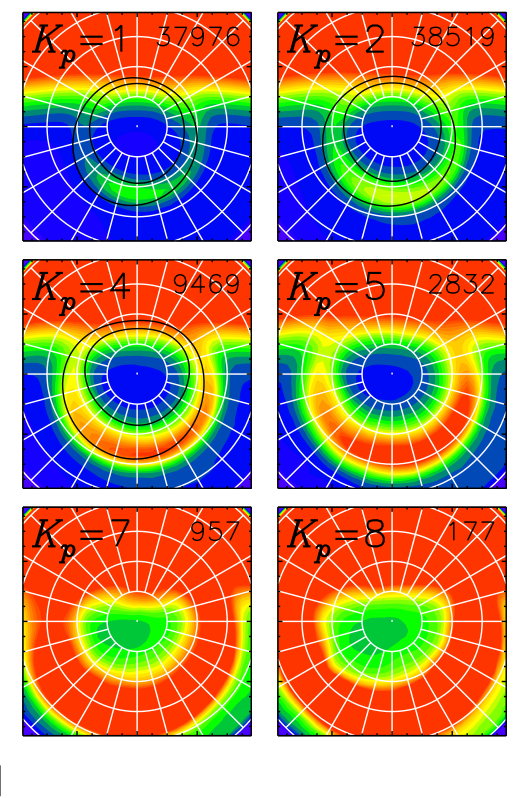

Fig. 1. Averaged SI12 (left) and WIC (right) observations of the Northern Hemisphere aurorae (observing period 2000 to 2002) parameterized by $K_{P}$, presented in a polar geomagnetic latitude and MLT frame, with local noon to the top of each panel. Concentric circles mark latitudes in $10^{\circ}$ steps, while radial white lines indicate MLT meridians. For $K_{P} \leqslant 4$ the appropriate Feldstein and Starkov (1967) average auroral oval is superimposed. The number in the top right of each panel indicates the number of individual images that were combined to produce the average. Elevated counts at the dayside are the result of dayglow.

of the magnetopause reconnection line, scaled from observations of the rate of expansion of the polar cap (Milan et al., 2008). Figure 3 shows average SI12 images binned according to solar wind velocity $V_{\mathrm{SW}}$ from 300 to $800 \mathrm{~km} \mathrm{~s}^{-1}$ in steps of $100 \mathrm{~km} \mathrm{~s}^{-1}$ (upper panels), solar wind density $N_{\mathrm{SW}}$ from 0 to $20 \mathrm{~cm}^{-3}$ in steps of $4 \mathrm{~cm}^{-3}$ (middle panels), and solar wind pressure $P_{\mathrm{SW}}$ from 0 to $15 \mathrm{nPa}$ in steps of $3 \mathrm{nPa}$ (lower panels); each of these has been further subdivided by IMF $B_{Z}$, such that $B_{Z}<0$ and $B_{Z}>0 \mathrm{nT}$. In each panel of Figs. 2 and 3, a Feldstein and Starkov (1967) model oval for $K_{P}=4$ has been superimposed to help guide the eye.

In each of the average images shown in Figs. 1 to 3, the maximum brightness in the nightside portion of the image, between 18:00 and 06:00 MLT, has been found; from this is subtracted the uniform background described above. The nightside of the images are isolated in this way to avoid contributions from dayglow, especially in the WIC images. Figure 4 presents the variation in the maximum brightness as a function of $K_{P}$ and solar wind parameters, SI12 in the top panels and WIC in the middle panels. In addition, the equivalent Southern Hemisphere results are shown in the bottom panels of Fig. 4. We also determined the total brightness in the nightside portions of the images and found dependencies that scale linearly with the results presented in Fig. 4, indicating that the variation in the maximum brightness is a good indicator of the variation in the total brightness. Furthermore, to quantify changes in the latitudinal location of the auroral oval, the latitude of the peak in brightness along the midnight meridian of each average image has been determined by fitting a Gaussian to the profile, and the results are indicated in Fig. 5.

Finally, as a check of the reproducibility of our results, and to determine if there are significant seasonal variations in the auroral response to the solar wind, as reported by Shue et al. (2002), we subdivided the Northern Hemisphere SI12 observations into "summer" and "winter" categories and redid our analysis. We included April, May, June, July, and August in the summer category and January, February, October, November and December in the winter category; hence, 10 months from two years of observations were included in each. The variations in auroral brightness and peak latitude are presented in Fig. 6, with summer results indicated in red and winter results in blue.

\section{Discussion}

Figure 1 shows the average auroral configuration associated with precipitating protons and electrons for different levels of $K_{P}$. As $K_{P}$ increases the auroral oval becomes brighter, increases in latitudinal width and the equatorward edge extends to lower latitudes. We note that the average oval is probably wider in latitude than would be the case in individual 

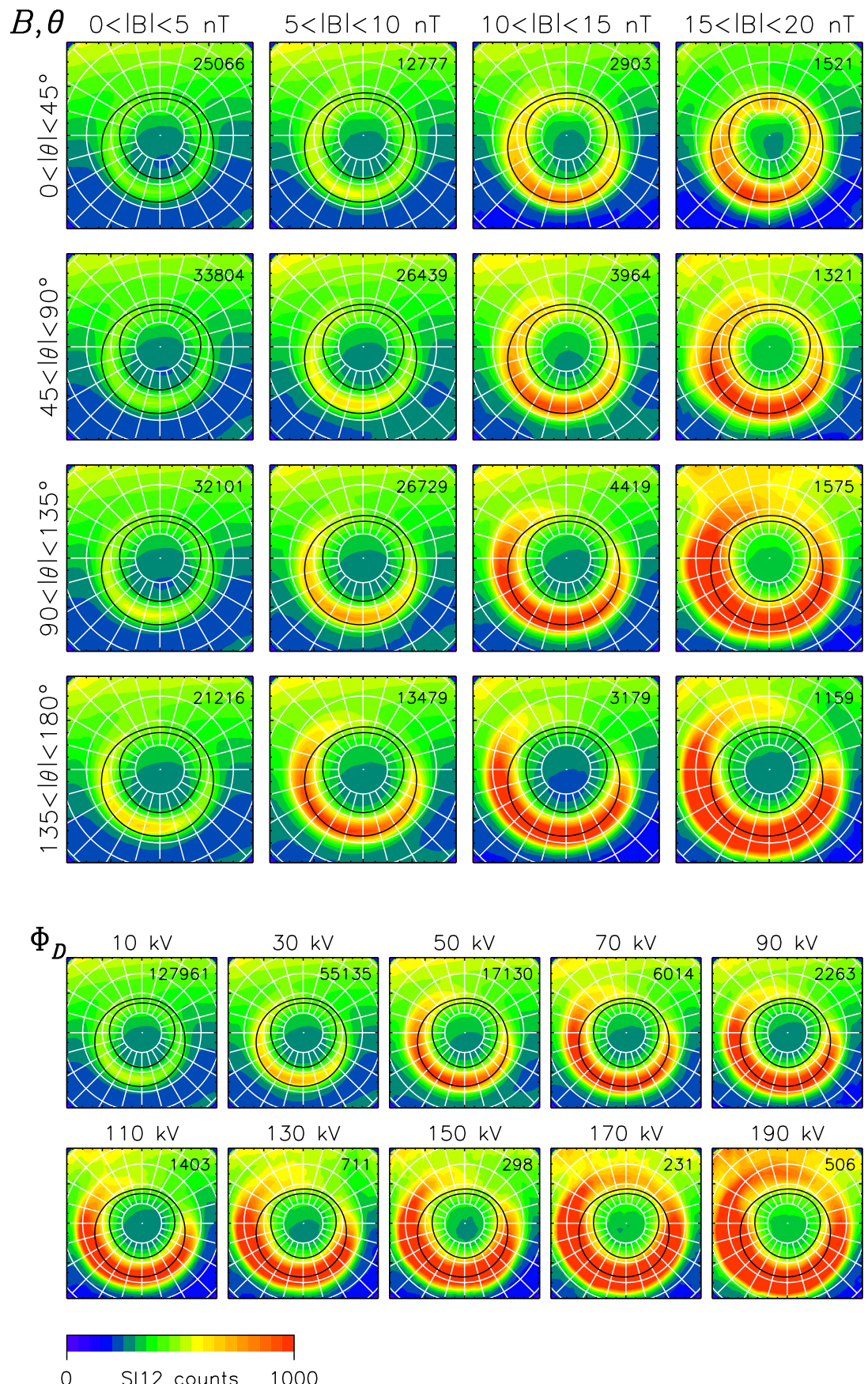

0

Fig. 2. Averaged SI12 observations parameterized by IMF magnitude and clock angle (top panels) and estimated dayside reconnection rate (bottom panels).

images due to variations in oval location within the $K_{P}$ bins. The auroral oval has a dawn-dusk asymmetry that is opposite for precipitating protons and electrons, being brighter in the dusk sector for protons, with a minimum in brightness near 09:00 MLT, and brighter in the dawn sector for electrons, with a minimum near 15:00 MLT. As noted by Mende et al. (2003), Boakes et al. (2008), and Milan et al. (2009) there is also a dawn-dusk offset in the locations of the proton and electron aurorae, protons being located at lower latitudes than electrons in the dusk sector and vice versa in the dawn sector. This can be seen most easily by comparing the locations of the proton and electron aurorae in the dawn and dusk sectors in the $K_{P}=4$ panels. These results are consistent with the offset seen in the locations and flux of precipitating 

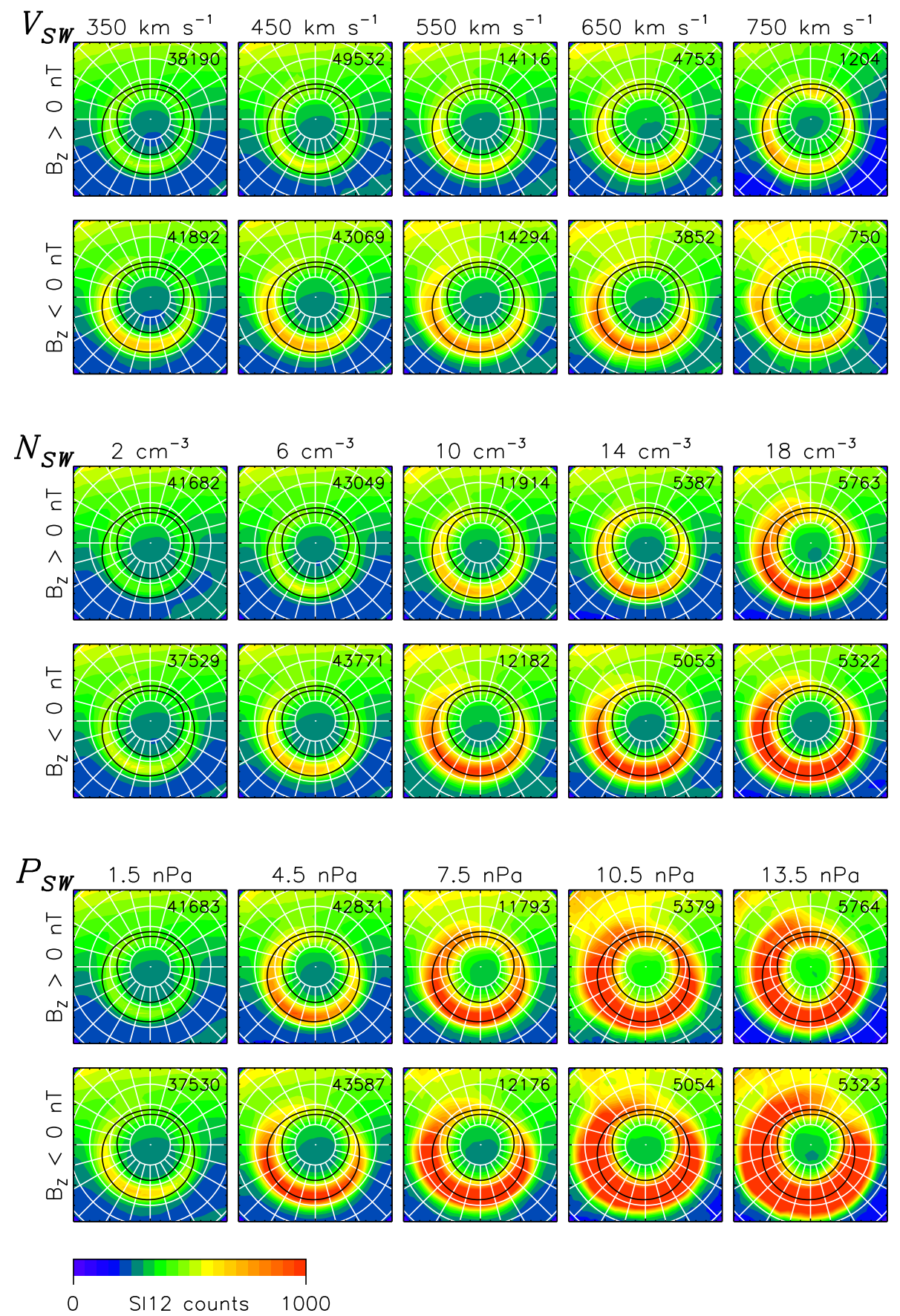

Fig. 3. Upper panels: Averaged SI12 observations parameterized by solar wind velocity. (Middle panels) Averaged SI12 observations parameterized by solar wind density. Bottom panels: Averaged SI12 observations parameterized by solar wind pressure. In each case the results are sub-divided for northward and southward IMF.

ions and electrons, as reported previously by Hardy et al. (1985, 1989) and Gussenhoven et al. (1987). As discussed by Gussenhoven et al. (1987) and reiterated most recently by Milan et al. (2009), the asymmetry in shape and intensity may be associated with the directions that protons and electrons drift around the Earth after being injected into the inner magnetosphere from the magnetotail, and the latitude offset may be associated with the relative locations of upwards and downwards field-aligned currents (FACs) in the region 1 and 2 current systems. The maximum auroral intensity varies by a factor of $\sim 25$ (SI12) or $\sim 70$ (WIC) from $K_{P}=0$ to $K_{P}=8$ (Fig. 4), with a similar variation in the total intensity. We 

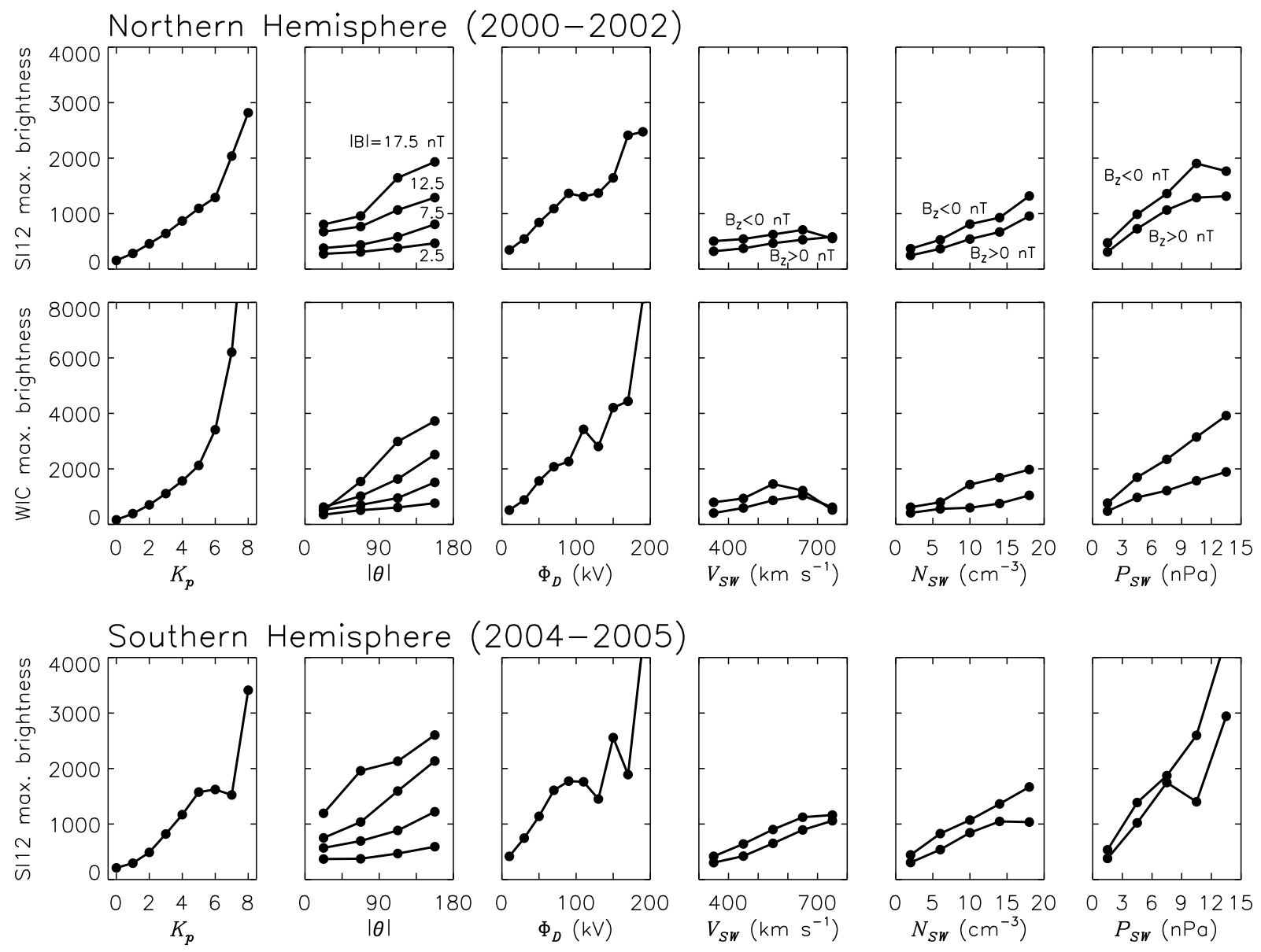

Fig. 4. The variation in the maximum nightside auroral intensity, parameterized by $K_{P}$, IMF magnitude and clock angle, estimated dayside reconnection rate, solar wind speed, density, and pressure. Upper panels: SI12 observations from the Northern Hemisphere (observing period 2000 to 2002). (Middle panels) WIC observations from the Northern Hemisphere. Bottom panels: SI12 observations from the Southern Hemisphere (observing period 2004 to 2005).

note that at the highest $K_{P}$ levels the number of contributing images becomes very small, especially for WIC, leading to increased uncertainties in the mean values of the intensity. Such uncertainties will be discussed in more detail below.

Figure 2 demonstrates that the proton aurorae become brighter and extend to lower latitudes as the total IMF magnitude $|B|$ increases and as the IMF orientation rotates from northwards $\theta=0^{\circ}$ to southwards $\theta=180^{\circ}$. The proton oval tends to be more symmetrical for northwards IMF, the dawndusk asymmetry becoming more pronounced for southward IMF. The proton oval also closely resembles the shape of the Feldstein and Starkov (1967) model for northward IMF, but extends to lower latitudes on the dayside for southward IMF. We also note that for near-zero clock angles and $|B| \geqslant 10 \mathrm{nT}$ an auroral cusp spot becomes apparent in the noon-sector oval. This cusp spot is associated with particle precipitation from a magnetopause reconnection site tailward of the cusp opening, otherwise known as lobe reconnection, during northward IMF (Milan et al., 2000; Frey et al., 2002; Fuselier et al., 2002). In the electron aurorae (not shown), fewer observations at high IMF magnitudes means that the mean ovals are somewhat less well-defined, but the variation in brightness and latitudinal extent is still clear, resembling that of the proton aurorae. Turning to Fig. 4, we note that the maximum (and total) SI12 brightness increase by a factor of $\sim 2-4$ as the IMF rotates from $\theta=0^{\circ}$ to southwards $\theta=180^{\circ}$, and by a factor of $\sim 5$ as the IMF magnitude increase from $|B|=2.5$ to $17.5 \mathrm{nT}$, for both electrons and protons. Overall, there is a factor of $\sim 10-15$ variation in brightness from low magnitude northward IMF to high magnitude southward IMF. In the case of WIC this is more pronounced, the variation being close to $\sim 30$. The reduced variation in SI12 brightness with respect to WIC brightness has been noted by previous workers (e.g., Milan et al., 2009) and is interpreted as resulting from the greater efficiency of pitch angle scattering of protons into the loss cone than electrons in the stretched 

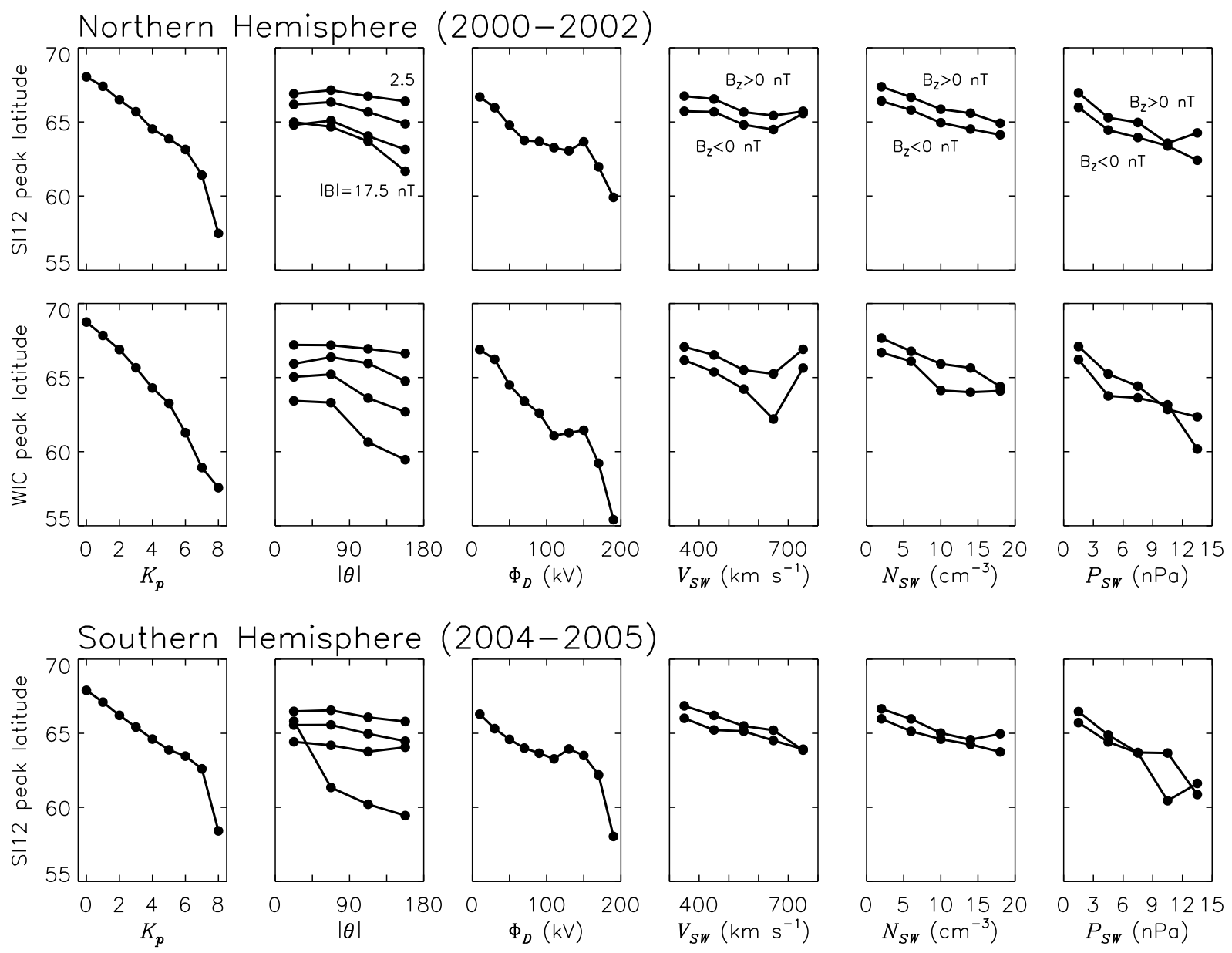

Fig. 5. Similar to Fig. 4, indicating the latitude of the peak in auroral brightness along the midnight meridian.

magnetic field lines of the magnetotail (e.g., Sergeev et al., 1983; Blockx et al., 2005). Hence, the precipitating proton flux remains relatively high, even at low levels of geomagnetic activity.

The variation in brightness and configuration of the SI12 oval with $\Phi_{D}$ is presented in the bottom panels of Fig. 2 . As $\Phi_{D}$ increases from $10 \mathrm{kV}$, the proton aurorae increase in brightness and expand to lower latitude. By $100 \mathrm{kV}$, the proton aurorae have taken on their familiar asymmetric shape, with a minimum in brightness at 09:00 MLT. Increasing further towards $200 \mathrm{kV}$, the oval becomes more symmetrical, extending to lower latitudes on the dayside and the 09:00 MLT gap filling in. A similar behaviour is observed in the electron aurorae (not shown), though with the minimum in brightness occurring at 15:00 MLT, and with the dayside dynamics largely obscured by dayglow. Turning to Fig. 4, a dramatic increase in brightness with $\Phi_{D}$ is observed, for both SI12 and WIC, with the increase rivalling that of the $K_{P}$ curves. There are some apparently random variations superimposed on these curves above values of $\Phi_{D}$ of $130 \mathrm{kV}$ or so, which we attribute to the relatively low numbers of images contributing to the means under these rare solar wind conditions. Clearly, $\Phi_{D}$ parameterizes the auroral response well, nearly as well as local measurements of geomagnetic disturbance.

The variations in SI12 auroral configuration and brightness with solar wind velocity, density, and pressure are shown in Fig. 3. Of most note is the increase in brightness with all three parameters (see also Fig. 4). The southward IMF cases are brighter than the corresponding northward IMF cases, as expected from the previous discussion. There are insufficient WIC observations in the highest $V_{\mathrm{SW}}$ bin to allow a comparison with SI12. Figure 4 indicates that the variation in maximum brightness is small for $V_{\mathrm{SW}}$, a factor of $\sim 2$ when $V_{\mathrm{SW}}$ varies between 350 and $750 \mathrm{~km} \mathrm{~s}^{-1}$, and a further factor of $\sim 2$ when the difference between IMF northward and southward is taken into account (the downturn in WIC brightness at high solar wind velocities should be discounted due to the insignificant number of observations). $N_{\text {SW }}$ orders the observations somewhat better, with a variation of a factor of $\sim 3$ as $N_{\mathrm{SW}}$ varies between 2 and $18 \mathrm{~cm}^{-3}$. 

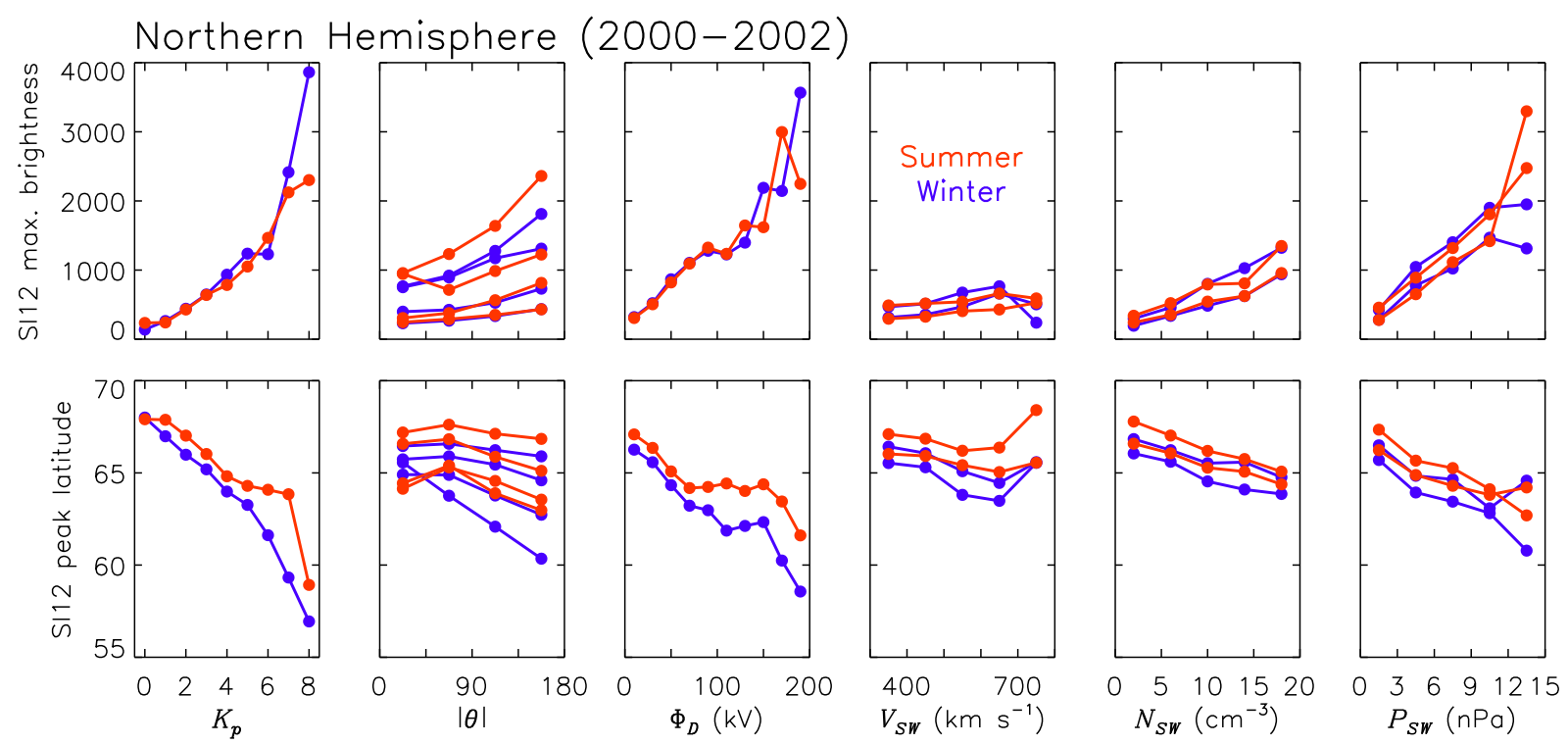

Fig. 6. Similar to Figs. 4 and 5, indicating the variation in the SI12 maximum nightside auroral intensity and the latitude of the peak in auroral brightness for the Northern Hemisphere observations (2000 to 2002), subdivided into summer months (red) and winter months (blue); see text for details.

$P_{\mathrm{SW}}$ orders the brightness rather better, with a variation of $\sim 5$ as $P_{\mathrm{SW}}$ varies between 1 and $15 \mathrm{nPa}$.

A proton cusp spot is apparent in the average image for $V_{\mathrm{SW}}=750 \mathrm{~km} \mathrm{~s}^{-1}$ and $B_{Z}>0 \mathrm{nT}$ (Fig. 3). No cusp spot is seen when the images are ordered by $N_{\mathrm{SW}}$. Frey et al. (2002) reported that the brightness of the cusp spot is modulated by the density of the solar wind, so our last finding is perhaps surprising. We suggest that if other solar wind conditions, for instance speed and IMF orientation, are favourable for the generation of a cusp spot then solar wind density will modulate this cusp spot brightness; however, high solar wind density is not a sufficient condition in itself to produce a cusp spot. We also note that the dayside auroral oval is generally brighter and thicker for high solar wind pressure than for high solar wind velocity or density.

Turning to Fig. 5, we now examine the changes in the latitude of the auroral oval in response to the geomagnetic and solar wind activity. We expect changes in latitude to be related to changes in the open magnetic flux content of the magnetosphere, i.e. the proportion of the terrestrial dipole that has been interconnected with the IMF by dayside reconnection (e.g., Milan et al., 2003). As the amount of open flux increases, the auroral oval should migrate equatorwards, and indeed we see a decrease in oval latitude as activity increases. In fact, we see a very similar variation in latitude (Fig. 5) as in oval brightness (Fig. 4). The variation in latitude is smallest when the data are binned by solar wind speed and density. A more significant variation is seen when the data are binned by solar wind pressure or with IMF clock angle and magnitude. Finally, the most significant variations are seen when the data are binned by $K_{P}$ or $\Phi_{D}$. The latter might be expected to order the observations well, as it is an estimate of the dayside reconnection rate, which should directly influence the amount of open magnetic flux in the magnetosphere. However, we note a plateau in the variation of latitude with $\Phi_{D}$ for values of $\Phi_{D}$ between 100 and $150 \mathrm{kV}$; it is possible that a similar plateau is present in the brightness binned by $\Phi_{D}$. It would be interesting to investigate if this plateau is the auroral counterpart of the saturation of the polar cap potential that has been reported in previous studies (e.g., Siscoe et al., 2002).

Shue et al. (2002) examined the dependence of the auroral brightness, as measured by Polar UVI, on $N_{\mathrm{SW}}, V_{\mathrm{SW}}$, and $P_{\mathrm{SW}}$. They found a significant difference in the responses for northward and southward IMF cases, especially in the slopes of the responses, which were greater for southward IMF. In our study, although the northward IMF auroral brightness tends to be lower than for southward IMF, the slope of the response to the solar wind parameters is similar for both (see Fig. 4). One possible explanation for this discrepancy is that Shue et al. investigated the changes in auroral brightness within specific bins in latitude and MLT, whereas our study concentrates on the change in peak brightness irrespective of the latitude at which it occurs. The results of Shue et al. will be influenced by the changing location of the auroral oval with $N_{\mathrm{SW}}, V_{\mathrm{SW}}$, and $P_{\mathrm{SW}}$ (Fig. 5), and do not straightforwardly reflect changes in brightness associated with these parameters.

Shue et al. (2002) also noted a significant difference in the auroral response to solar wind conditions depending on 
season, the auroral brightness increasing more steeply in winter than in summer. We investigate this also by subdividing the Northern Hemisphere SI12 observations into summer and winter months, presented in Fig. 6. We find in general no significant differences between the summer and winter brightness responses; where there are differences, these can be attributed to low numbers of images contributing to the means. This result gives us confidence in the reproducibility of our findings. The variations in the latitude of the aurorae are very similar for summer and winter months; however, we find a systematic offset of between $1^{\circ}$ and $2^{\circ}$ of latitude between the summer and winter curves, the winter curves being displaced to lower latitudes.

\section{Conclusions}

Creating average images of the auroral oval has allowed us to investigate the solar wind influence on auroral intensity and configuration for varying upstream solar wind conditions. On the whole, the observations show that the variations in the brightness and latitude of the auroral oval are best ordered by $K_{P}$, which is intuitive as the magnetic perturbations measured by $K_{P}$ are directly associated with conductivity enhancements produced by auroral precipitation. Having said this, the predicted dayside reconnection rate $\Phi_{D}$, related to the solar wind electric field, orders the observations almost as well. Intriguingly, there appears to be a plateau in the auroral response to $\Phi_{D}$ between values of 100 and $150 \mathrm{kV}$. It is not clear what causes this, though it is possible that it relates to a change in the mode of response of the magnetosphere to increasing solar wind driving, or the reported saturation of the transpolar voltage for strong solar wind driving (e.g., Siscoe et al., 2002). This will be examined in more detail in a subsequent study.

Examining the influence of individual solar wind properties such as speed, density, or the embedded IMF, we find that variations in the IMF are most important, not only in the variation in brightness, but also in controlling the configuration of the aurorae, such as the presence of a northward IMF cusp spot. Solar wind pressure orders the data better than solar wind density, and solar wind velocity has the least impact. It should be noted that although $N_{\mathrm{SW}}$ has a more significant impact than $V_{\mathrm{SW}}$ on the auroral brightness, it is not included in the calculation of $\Phi_{D}$, and it is possible that a solar wind-magnetosphere coupling function that includes a contribution from $N_{\mathrm{SW}}$ may improve on the performance of $\Phi_{D}$ (e.g., Newell et al., 2007).

Acknowledgements. TAE was supported by a Nuffield Undergraduate Bursary, for which we would like to thank the Nuffield Foundation. The IMAGE FUV data were supplied by the NASA Space Science Data Centre (NSSDC), and we are grateful to the PI of FUV, S. B. Mende of the University of California at Berkeley, for its use. The ACE data used in this study were accessed through CDAWeb. The authors would like to thank N. F. Ness at the Bartol Research
Institute and D. J. McComas of the Southwest Research Institute, for use of the MAG and SWEPAM data, respectively.

Topical Editor I. A. Daglis thanks J. Burch and another anonymous referee for their help in evaluating this paper.

\section{References}

Blockx, C., Gérard, J.-C., Meurant, M., Hubert, B., and Coumans, V.: Far ultraviolet remote sensing of the isotropy boundary and magnetotail stretching, J. Geophys. Res., 110, A11215, doi:10.1029/2005JA011103, 2005.

Boakes, P. D., Milan, S. E., Abel, G. A., Freeman, M. P., Chisham, G., Hubert, B., and Sotirelis, T.: On the use of IMAGE FUV for estimating the latitude of the open/closed magnetic field line boundary in the ionosphere, Ann. Geophys., 26, 2759-2769, 2008 ,

http://www.ann-geophys.net/26/2759/2008/.

Burch, J. L.: IMAGE mission overview, Space Sci. Rev., 91, 1-14, 2000.

Coumans, V., Gérard, J.-C., Hubert, B., Mende, S. B., and Cowley, S. W. H.: Morphology and seasonal variations of global auroral proton precipitation observed by IMAGE-FUV, J. Geophys. Res., 109, A12205, doi:10.1029/2003JA010348, 2004.

Coumans, V., Gérard, J.-C., Hubert, B., and Meurant, M.: Global auroral proton precipitation observed by IMAGE-FUV: Noon and midnight brightness dependence on solar wind characteristics and IMF orientation, J. Geophys. Res., 111, A05210, doi:10.1029/2005JA011317, 2006.

Coumans, V., Blockx, C., Gérard, J.-C., Hubert, B., and Connors, M.: Global morphology of substorm growth phases observed by the IMAGE-SI12 imager, J. Geophys. Res., 112, A11211, doi:10.1029/2007JA012329, 2007.

Fairfield, D. H.: Polar magnetic disturbances and the interplanetary magnetic field, Space Res., VIII, 107-119, 1967.

Feldstein, Y. I. and Starkov, G. V.: Dynamics of auroral belt and geomagnetic disturbances, Planet. Space Sci., 15, 209-229, 1967.

Frank, L. A. and Craven, J. D.: Imaging results from Dynamics Explorer 1, Rev. Geophys., 26, 249-283, 1988.

Frey, H. U., Mende, S. B., Immel, T. J., Fuselier, S. A., Claflin, E. S., Gérard, J.-C., and Hubert, B.: Proton aurora in the cusp, J. Geophys. Res., 107, A1091, doi:10.1029/2001JA900161, 2002.

Frey, H. U., Mende, S. B., Immel, T. J., Gérard, J.-C., Hubert, B., Habraken, S., Spann, J., Gladstone, G. R., Bisikalo, D. V., and Shematovich, V. I.: Summary of quantitative interpretation of IMAGE Far Ultraviolet auroral data, Space Sci. Rev., 109, 255283, 2003.

Fuselier, S. A., Frey, H. U., Trattner, K.-H., Mende, S. B., and Burch, J. L.: Cusp aurora dependence on interplanetary magnetic field $B_{Z}$, J. Geophys. Res., 107, A1111, doi:10.1029/2001JA900165, 2002.

Gussenhoven, M. S., Hardy, D. A., and Heinemann, N.: The equatorward boundary of auroral ion precipitation, J. Geophys. Res., 92, 3273-3283, 1987.

Hardy, D. A., Gussenhoven, M. S., and Holeman, E.: A statisticalmodel of auroral electron-precipitation, J. Geophys. Res., 90, 4229-4248, 1985.

Hardy, D. A., Gussenhoven, M. S., and Brautigam, D.: A statistical model of auroral ion precipitation, J. Geophys. Res., 94, 370 392, 1989. 
Heppner, J. P. and Maynard, N. C.: Empirical high-latitude electricfield models, J. Geophys. Res., 92, 4467-4489, 1987.

Holzworth, R. H. and Meng, C.-I.: Mathematical representation of the auroral oval, Geophys. Res. Lett., 2, 377-380, 1975.

Kan, J. R. and Lee, L. C.: Energy coupling function and solar windmagnetosphere dynamo, Geophys. Res. Lett., 6, 577-580, 1979.

Liou, K., Newell, P. T., Meng, C.-I., Brittnacher, M., and Parks, G.: Characteristics of the solar wind controlled auroral emissions, J. Geophys. Res., 103, 17543-17557, 1998.

McComas, D. J., Bame, S. J., Barker, P., Feldman, W. C., Phillips, J. L., Riley, P., and Griffee, J. W.: Solar Wind Electron Proton Alpha Monitor (SWEPAM) for the Advanced Composition Explorer, Space Sci. Rev., 86, 563-612, 1998.

Mende, S. B., Heetderks, H., Frey, H. U., Lampton, M., Geller, S. P., Habraken, S., Renotte, E., Jamar, C., Rochus, P., Spann, J., Fuselier, S. A., Gérard, J.-C., Gladstone, R., Murphree, S., and Cogger, L.: Far ultraviolet imaging from the IMAGE spacecraft. 1. System design, Space Sci. Rev., 91, 243-270, 2000a.

Mende, S. B., Heetderks, H., Frey, H. U., Lampton, M., Geller, S. P., Abiad, R., Siegmund, O. H. W., Tremsin, A. S., Spann, J., Dougani, H., Fuselier, S. A., Magoncelli, A. L., Bumala, M. B., Murphree, S., and Trondsen, T.: Far ultraviolet imaging from the IMAGE spacecraft. 2. Wideband FUV imaging, Space Sci. Rev., 91, 271-285, 2000b.

Mende S. B., Frey, H. U., Morsony, B. J., and Immel, T. J.: Statistical behavior of proton and electron auroras during substorms, J. Geophys. Res., 108, 1339, doi:10.1029/2002JA009751, 2003.

Milan, S. E., Lester, M., Cowley, S. W. H., and Brittnacher, M.: Dayside convection and auroral morphology during an interval of northward interplanetary magnetic field, Ann. Geophys., 18, 436-444, 2000, http://www.ann-geophys.net/18/436/2000/.

Milan, S. E., Lester, M., Cowley, S. W. H., Oksavik, K., Brittnacher, M., Greenwald, R. A., Sofko, G., and Villain, J.-P.: Variations in the polar cap area during two substorm cycles, Ann. Geophys., 21, 1121-1140, 2003, http://www.ann-geophys.net/21/1121/2003/.

Milan, S. E., Boakes, P. D., and Hubert, B.: Response of the expanding/contracting polar cap to weak and strong solar wind driving: implications for substorm onset, J. Geophys. Res., 113, A09215, doi:10.1029/2008JA013340, 2008.
Milan, S. E., Grocott, A., Forsyth, C., Imber, S. M., Boakes, P. D., and Hubert, B.: A superposed epoch analysis of auroral evolution during substorm growth, onset and recovery: open magnetic flux control of substorm intensity, Ann. Geophys., 27, 659-668, 2009, http://www.ann-geophys.net/27/659/2009/.

Newell, P. T., Sotirelis, T., Liou, K., Meng, C.-I., and Rich, F. J.: A nearly universal solar wind-magnetosphere coupling function inferred from 10 magnetospheric state variables, J. Geophys. Res., 112, A01206, doi:10.1029/2006JA012015, 2007.

Reiff, P., Spiro, R., and Hill, T.: Dependence of polar cap potential drop on interplanetary parameters, J. Geophys. Res., 86, 76397648, 1981.

Ruohoniemi, J. M. and Greenwald, R. A.: Statistical patterns of high-latitude convection obtained from Goose Bay HF radar observations, J. Geophys. Res., 101, 21743-21763, 1996.

Scurry, L. and Russell, C. T.: Proxy studies of energy transfer to the magnetosphere, J. Geophys. Res., 96, 9541-9548, 1991.

Sergeev, V. A., Sazhina, E. M., and Tsyganenko, N. A.: Pitch-angle scattering of energetic protons in the magnetotail current sheet as the dominant source of their isotropic precipitation into the nightside ionosphere, Planet. Space Sci., 31, 1147-1155, 1983.

Shue, J.-H., Newell, P. T., Liou, K., and Meng, C.-I.: Solar wind density and velocity control of auroral brightness under normal interplanetary magnetic field conditions, J. Geophys. Res., 107, 1428, doi:10.1029/2001JA009138, 2002.

Siscoe, G. L., Crooker, N. U., and Siebert, K. D.: Transpolar potential saturation: Roles of region 1 current system and solar wind ram pressure, J. Geophys. Res., 107, 1321, doi:10.1029/2001JA009176, 2002.

Smith, C. W., L'Heureux, J., Ness, N. F., Acuña, M. H., Burlaga, L. F., and Scheifele, J.: The ACE Magnetic Field Experiment, Space Sci. Rev., 86, 613-632, 1998.

Stone, E. C., Frandsen, A. M., Mewaldt, R. A., Christian, E. R., Marglies, D., Ormes, J. F., and Snow, F.: The Advanced Composition Explorer, Space Sci. Rev., 86, 1-22, 1998. 